\title{
ROF V/s Traditional Fiber as a Backhaul Technology: Comparisons of Cellular and Ethernet Networks based on SNR and Capacity
}

\author{
Rizwan lqbal \\ Department of Telecommunications \\ Mohammad Ali Jinnah University, Karachi
}

\begin{abstract}
Radio over Fiber is the future technology. It will take over all traditional fiber technology, as it offers quality and service up to the requirement of the users. The ROF is being implemented over traditional fiber widely as it offers replacing of optical fiber's high range and also flexible in wireless mode. Along with the ease, optical fiber bandwidth utilization increase by this. Because fiber is a high bandwidth, low latency, and a scalable solution therefore it is mostly used as a backbone technology in wireless Ethernet and cellular networks. Radio over Fiber technology, it is amalgamation of radio waves and optical based networks and it is an emergent feasible solution for providing high capacity and wireless connectivity, meanwhile reducing costs in the network which is accessible. The purpose of this research is to conduct a comparative study on implementation of ROF over wireless Ethernet network and cellular networks. Recently fiber optics has become a standard medium for these heterogeneous networks and introducing radio technology over this medium can prove to be the next generation solution. SNR and Capacity are the parameters that are critically evaluated for the purpose of comparative study on currently implemented fiber with ROF over these heterogeneous networks. Then comparative study is used to identify ROF is a feasible alternative as compared to traditional fiber as the backbone technology. A modeled ROF and optical fiber network is deployed over MATLAB for acquiring the desired results through simulation to prove the hypothesis. In future some other heterogeneous networks and several parameters like bit error rate and carrier to noise ratio can be analyzed on radio over fiber. A prototype of such an implementation will be proposed and design for small scale deployment to observe the behavior of radio over fiber in terms of aforementioned parameters.
\end{abstract}

\section{Keywords}

ROF, fiber optics, cellular network, Ethernet network, SNR and Capacity

\section{INTRODUCTION}

Radio over Fiber is the future technology. It will take over all traditional fiber technology, as it offers quality and service up to the requirement of the users. The ROF is being implemented over traditional fiber widely as it offers replacing of optical fiber's high range and also flexible in wireless mode. Along with the ease, optical fiber bandwidth utilization increase by this. Recently fiber optics has become a standard medium for Ethernet and cellular based networks and introducing radio technology over this medium can prove to be the next generation solution.
Following are the parameters that will be critically evaluated for the purpose of comparative study on currently implemented fiber with ROF over these heterogeneous networks:

- $\quad$ Signal to noise ratio

- Capacity

\section{TRADITIONAL FIBER}

Fiber optics infrastructure has blasted over the previous 2 decades. Fiber is an essential part of the current day transmission and the infrastructure of fiber optic can be installed on the roads, buildings and every those places where high bandwidth data rates are required. Fiber is made up of glass which based on silica. It look like a human hair and coated by transparent cladding. For the transmission of information on high data rates over a large distance, light will be transmitted on the fiber [1]. Today wide area of world approximately $80 \%$ long distance communication can transmit the data over optical fiber cables, millions of kilometers of cable has been installed all over the world. Now a day optical fiber cable with minimum loss offers infinite bandwidth and unique benefits over all transmission media which installed in past [2].

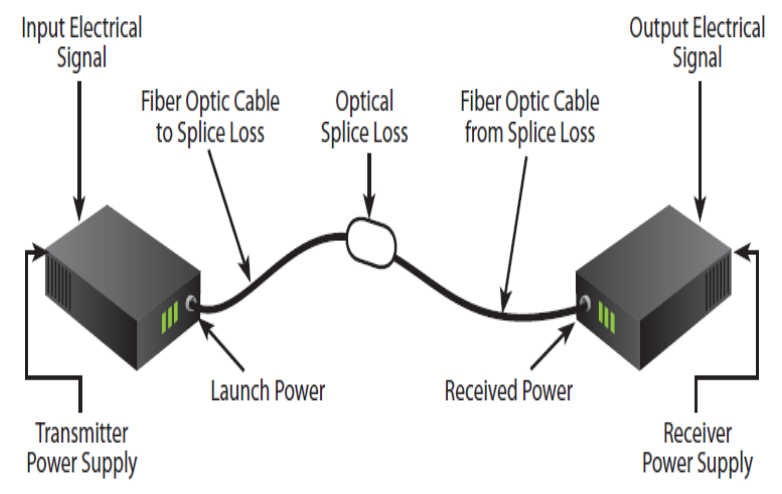

Fig 1: Optical fiber system

\section{RADIO OVER FIBER}

Radio over Fiber technology, it is amalgamation of radio waves and optical based networks and it is an emergent feasible solution for providing high capacity and wireless connectivity, meanwhile reducing costs in the network which is accessible. A radio over fiber link based on hardware need to set radio frequency signal at optical carrier, then provide connectivity of optical fiber and equipment needs radio frequency signal by the carrier. $1.3 \mathrm{~nm}$ spectrum normally selected to coincide for optical carrier's wavelength, in this SMF (single mode fiber) has low dispersion and in 1.5 
nm spectrum has low noise [3]. The remote antenna unit's Perform optoelectronic conversion and increase in amplitude celled amplification function. The advantages of the radio over fiber technology is differentiate with electronically distribution are easy installation, maintenance, decrease attenuation loss, increase bandwidth, reduced power consumption, free to RF interference [4]. There are many optical way outs of generating and transmitting microwave signals over fiber. The frequency of RF signal impose in to radio over fiber link at the top end in contrast with the signal initiate at the remote antenna unit. Radio frequency over fiber, base band over fiber and intermediate frequency over fiber are the main three categories by ROF can be categorized [5].

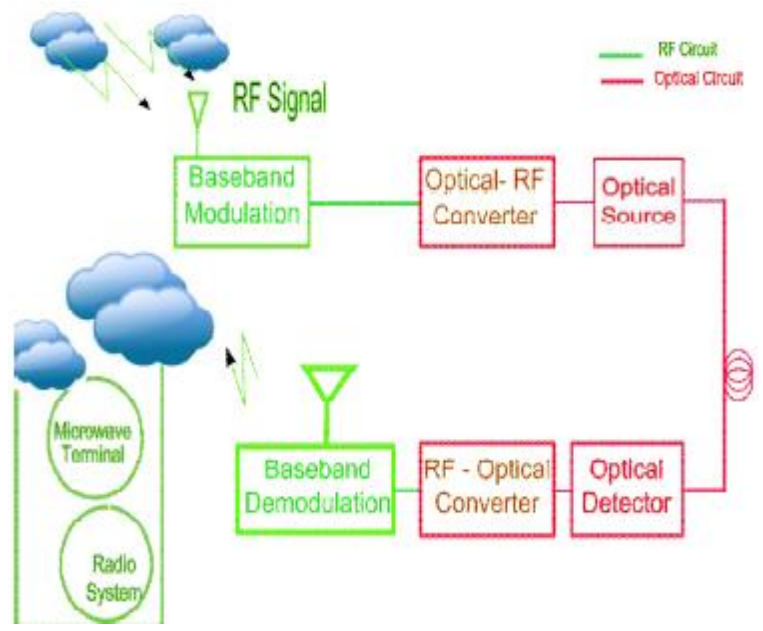

Fig 2: Block diagram of ROF

\section{ETHERNET NETWORKS}

In 1970 Ethernet transform by its primary conception. It was established specially for local area network to interconnect with each other, and it has grown to be the common wide home network and also corporate network technology. In 1990 optical interfaces were standardized by Ethernet. Most companies use Ethernet in the LANs for run heavy storage applications [6]. Because of this feature, the use of Ethernet in corporate world increases and production of Ethernet equipment decreases and standard of Ethernet has been permanently improved their speed [7]. Now a day gigabit Ethernet has developed for this purpose because mixed technology is able to decreasing the cost, and enterprises are increasingly demanding more Ethernet based environment. Ethernet systems also support CSMA/CD Protocol to provide a better accessibility of data or information. 100-BASET or fast Ethernet transmits 100 megabits/second speed provide for transmission, this standard usually used for Local Area Network backbone system and support 10-BASET cards with computer. 1000 megabits or Giga bit Ethernet supports an even higher level of LANs backbone system and it will support 1000 megabits or 1 gigabit or 1 billion bits/second as well as 10 gigabits support up to 10 billion bits per second [8].

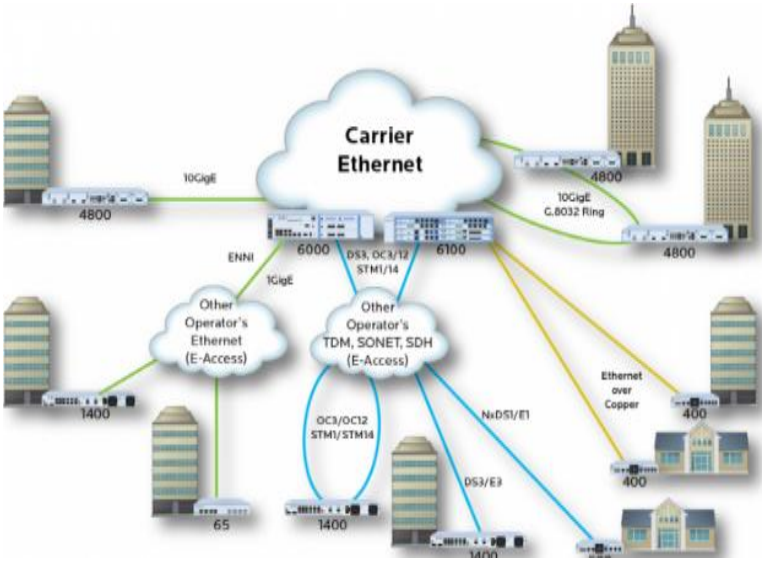

Fig 3: Ethernet Networks

\section{CELLULAR NETWORKS}

From past 2 decades, cellular communication explosively grown. Now a day millions of client's use cellular phone in all over the world. Cell phones permits the clients to transmit or received data in the form of signal from anywhere in the world. Mobility is the main factor for growing the industry of cellular network. With the integration of cellular phones and public switched telephone network (PSTN), appears a new system called cellular network system and this system supports cellular communication. Usually cellular network gives cell phones with no wire connection or wireless access to PSTN. Cell is the smallest covered area of cellular network, and combination of more than one cell make a large cellular network which provides the connectivity between different mobile stations with the help of base station (BS) [9]. The base station is static and directly connected to the mobile switching center (MSC); it is also called MTSO (mobile telephone switching office). An MSC depends on the various BSs (base stations) and directly connected to the public switched telephone network. Mobile stations and base stations are consists of transceivers, limited frequency spectrum are allocate for this network [10].

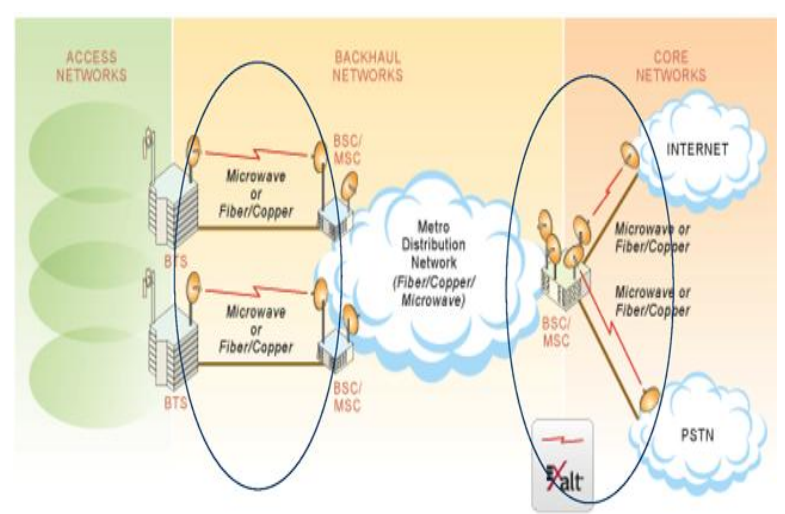

Fig 4: Cellular Networks

A modeled ROF network is deployed over MATLAB for acquiring the desired results to prove the hypothesis. After this comparison ROF is critically evaluated whether it is feasible alternative as compared to traditional fiber as the backbone technology.

\section{ANALYSIS}

This section defines the analytical approach of both technologies i.e. fiber optic and radio over fiber implemented in two different environments that are cellular network and 
Ethernet network. The two technologies are examined on the following parameters in above mentioned environments.

\subsection{Signal to Noise Ratio (SNR)}

$\mathrm{S} / \mathrm{N}$ is the ratio of the strength of electrical or other signal carrying information to that of unwanted interference. It is measures in dbs.

\subsubsection{Cellular Network}

The following equation is used to develop a graph for Signal to Noise Ratio in cellular network when traditional fiber works as backhaul technology.

$$
\mathrm{SNR}_{(\mathrm{OFC})}=2 * \log (\mathrm{L}) *(\mathrm{P} 0-\mathrm{Pin}) / \mathrm{NA} * \mathrm{D} * \mathrm{r} 0
$$

Where;

$\mathrm{SNR}_{(\mathrm{OFC})}$ : Signal to Noise Ratio

L: Length of the fiber optic in $\mathrm{km}$

P0: Laser pulse power in $\mathrm{mW}(0.1$ to $10 \mathrm{~mW})$

Pin: Input Power 10mW

NA: Numerical Aperture

D: Dispersion parameter in $\mathrm{ps} / \mathrm{nm} . \mathrm{km}$

$\mathrm{r} 0$ : fiber spot size in $\mathrm{m}^{\wedge} 2$

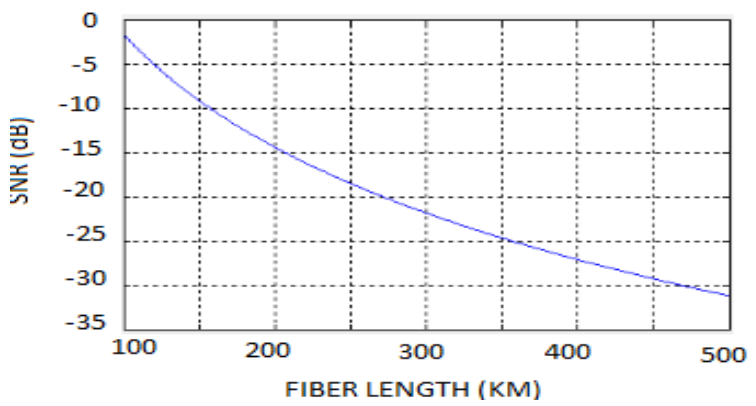

Fig 5: Signal to noise ratio of traditional fiber in cellular network

Figure 5 shows that signal to noise ratio is decreases with increase in length of fiber. When traditional fiber used as a backhaul technology in cellular network, signal to noise ratio decreases with increase in the length of fiber. The range of fiber length is 100 to $500 \mathrm{~km}$.

The following equation is used to develop a graph for Signal to Noise Ratio in cellular network when ROF works as backhaul technology.

$$
\mathrm{SNR}_{(\mathrm{ROFC})}=2 * \log (\mathrm{L}+\mathrm{NA}-\mathrm{Tp}) *(\mathrm{P} 0-\mathrm{Pin}) / \mathrm{NA} * \mathrm{D}+\mathrm{n} 2 * \mathrm{r} 0
$$

Where,

SNR ${ }_{(\mathrm{ROFC})}$ : Signal to Noise Ratio

Tp: Pulse time (width) in seconds

$\mathrm{n} 2$ : Non-linear index coefficient in $\mathrm{m}^{\wedge} 2 / \mathrm{W}$

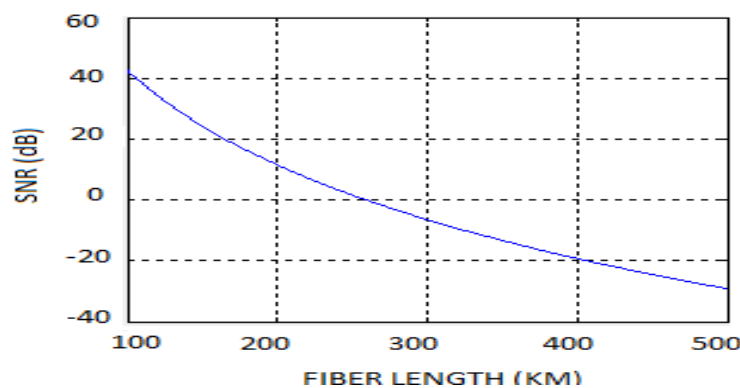

Fig 6: Signal to Noise Ratio of ROF in cellular network

Figure 6 shows that radio over fiber used as a backhaul technology in cellular network, the signal to noise ratio decreases with increases in fiber length. Signal to noise ratio is decreasing as the length of fiber increase from 100 to $500 \mathrm{~km}$.

Both graphs show the relationship between fiber lengths in $\mathrm{km}$ and signal to noise ratio in decibel. In graph $\mathrm{x}$-axis is represented by fiber length and y-axis is represented by signal to noise ratio. With the help of above simulated graphs we analyze that radio over fiber gives maximum SNR as compared to traditional fiber in cellular environment.

\subsubsection{Ethernet Network}

The following equation is used to develop a graph for Signal to Noise Ratio in Ethernet network when traditional fiber works as backhaul technology.

$$
\mathrm{SNR}_{(\mathrm{OFE})}=2 * \log (\text { Fiber stream }+\mathrm{Tp})+\mathrm{P} 0 \text {-Pin }
$$

Where;

SNR $_{(\mathrm{OFE})}$ : Signal to Noise Ratio

Fiber Stream: Fiber Data

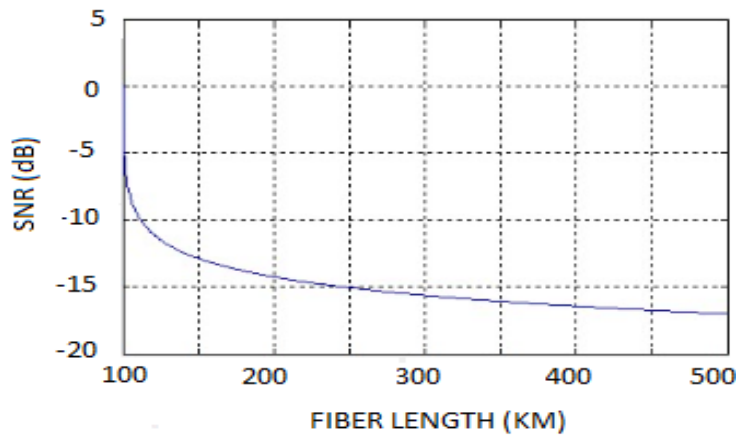

Fig 7: Signal to Noise Ratio of traditional fiber in Ethernet network

Figure 7 shows that signal to noise ratio decreases with increase in fiber length. Initially Signal to Noise Ratio decreases rapidly after rapid decrement Signal to Noise Ratio decreases gradually with increase in the fiber length. It means when fiber length increase from 100 to $500 \mathrm{~km} \mathrm{SNR}$ decreases from $-5 \mathrm{~dB}$ to $-17 \mathrm{~dB}$.

The following equation is used to develop a graph for Signal to Noise Ratio in Ethernet network when ROF works as backhaul technology.

\section{$\mathrm{SNR}_{(\mathrm{ROFE})}=(\mathrm{P} 0-\mathrm{Pin}) / \mathrm{NA} * \mathrm{D}+\mathrm{n} 2 * \mathrm{r} 0+2 * \log (\mathrm{ROF}$ stream $)$}

Where;

$\mathrm{SNR}_{(\mathrm{ROFE})}$ : Signal to Noise Ratio ROF stream: ROF data

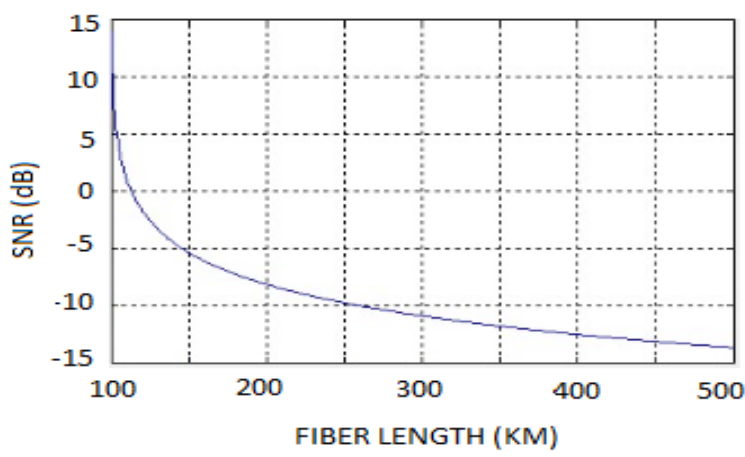

Fig 8: Signal to Noise Ratio of ROF in Ethernet network

Figure 8 shows Signal to Noise Ratio decreases with increase in fiber length. Initially rapid decrement in Signal to Noise 
Ratio but after rapid decrement Signal to Noise Ratio decreases gradually with increase in the fiber length. The range of fiber length is 100 to $500 \mathrm{~km}$ and value of Signal to Noise Ratio is $10 \mathrm{db}$ to $-14 \mathrm{db}$.

Both graphs shows the relationship between fiber length in $\mathrm{km}$ and Signal to Noise Ratio in decibel. In graph $\mathrm{x}$-axis is represented by fiber length and $y$-axis is represented by Signal to Noise Ratio. With the help of above simulated graphs we analyze that radio over fiber gives maximum SNR as compared to traditional fiber in Ethernet environment.

\subsection{Capacity}

Capacity can be defined as the maximum amount which can hold by a system, as a backbone link it is refer to no. of users.

\subsubsection{Cellular Network}

The following equation is used to develop a graph for capacity in cellular network when traditional fiber works as backhaul technology.

$$
\begin{aligned}
\mathrm{CAP}_{(\mathrm{OFC})}= & \operatorname{sqrt}((\text { deltaz+iterations+r0-n2))+ } \\
& \left(\cos \left(\mathrm{D} /\left(\text { time }^{*} \mathrm{Tp}\right)\right)\right)
\end{aligned}
$$

Where;

$\mathrm{CAP}_{(\mathrm{OFC})}$ : Capacity

Deltaz: Split step length in km

Iterations: Length of the fiber optic in $\mathrm{km}(\mathrm{L}) /$ split step length in $\mathrm{km}$ (deltaz)

r0: Fiber spot size in $\mathrm{m}^{\wedge} 2$

$\mathrm{n} 2$ : Non-linear index coefficient in $\mathrm{m}^{\wedge} 2 / \mathrm{W}$

D: Dispersion parameter in ps/nm.km

Time: simulation time for desired bit string

Tp: Pulse time (width) in seconds

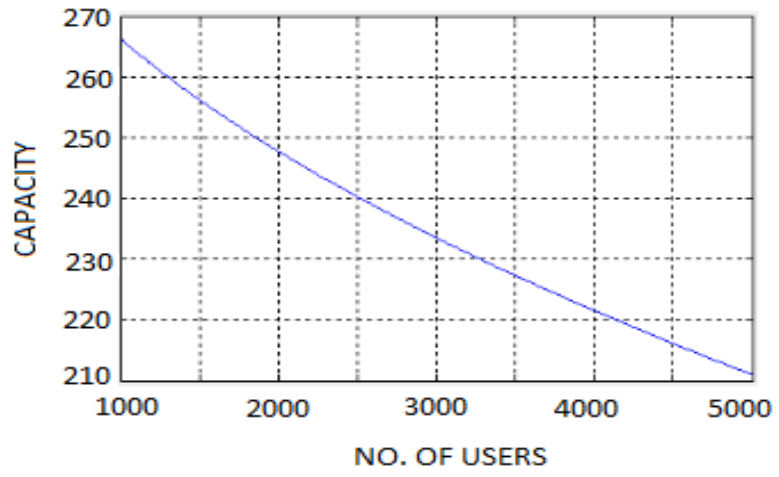

Fig 9: Capacity of traditional fiber in cellular network

Figure 9 shows linear decrement in capacity when no. of users increases from 1000 to 5000. It shows capacity decreases in cellular network when optical fiber used as a backhaul technology.

The following equation is used to develop a graph for capacity in cellular network when radio over fiber works as backhaul technology.

Where;

$$
\begin{aligned}
\mathrm{CAP}_{(\mathrm{ROFC})}=\left(\left(\mathrm{cld}+\mathrm{cd}^{*} \text { iterations }+\mathrm{r} 0-\mathrm{n} 2+\mathrm{ROF}\right.\right. \\
\text { stream }))-(\cos (\mathrm{D} / \mathrm{time}))
\end{aligned}
$$

$\mathrm{CAP}_{(\mathrm{ROFC})}$ : Capacity

cld: Cladding Diameter for Single Mode Fiber

cd: Core Diameter for Optical Fiber

ROF stream: ROF Data

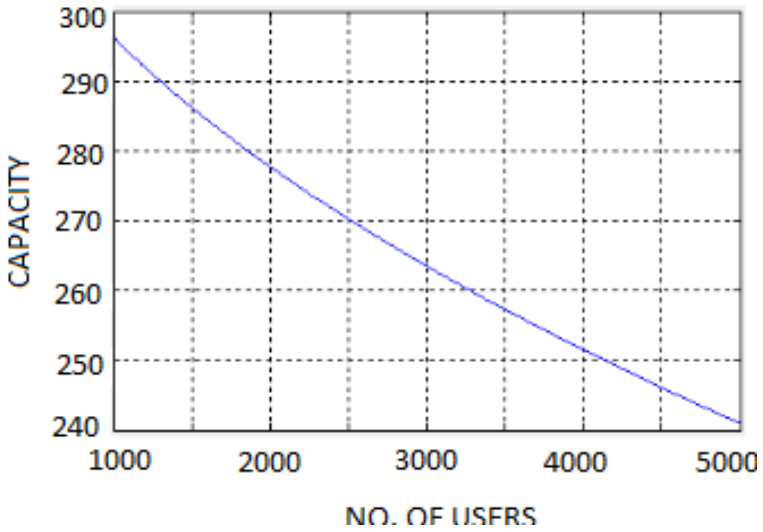

Fig 10: Capacity of ROF in cellular network

Figure 10 shows linear decrement in capacity when fiber length increases from 100 to $500 \mathrm{~km}$.

After analysis of both graphs which represents the relationship between no. of users on $x$-axis and capacity on $y$-axis, we conclude the result that capacity gives better performance when radio over fiber used as a backbone technology in cellular networks.

\subsubsection{Ethernet Network}

The following equation is used to develop a graph for capacity in Ethernet network when traditional fiber works as backhaul technology.

Where;

$$
\begin{aligned}
\mathrm{CAP}_{(\mathrm{OFE})} & =\operatorname{sqrt}((\mathrm{cld}+\mathrm{cd} * \text { deltaz }+\mathrm{r} 0+\mathrm{c})) * \text { Fiberstream } \\
& +(\cos (\mathrm{D} / \text { time }))
\end{aligned}
$$

CAP (OFE): Capacity

c: speed of light in $\mathrm{m} / \mathrm{s}$

Fiber stream: Fiber Data

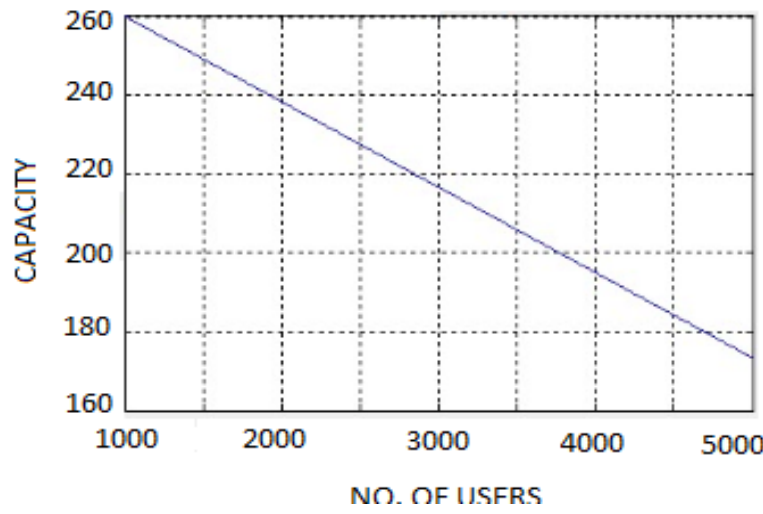

Fig 11: Capacity of traditional fiber in Ethernet network

Figure 11 shows that capacity is inversely proportional to no. of users when traditional fiber deployed as a backhaul technology in Ethernet network.

The following equation is used to develop a graph for capacity in Ethernet network when radio over fiber works as backhaul technology.

Where;

$$
\begin{aligned}
\mathrm{CAP}_{(\mathrm{ROFE})} & =\operatorname{sqrt}((\mathrm{cld}+\mathrm{cd} * \text { deltaz+r0 })) * \text { ROFstream } \\
& +(\cos (\mathrm{D} / \text { time }))
\end{aligned}
$$

$\mathrm{CAP}_{(\mathrm{ROFE})}$ : Capacity 


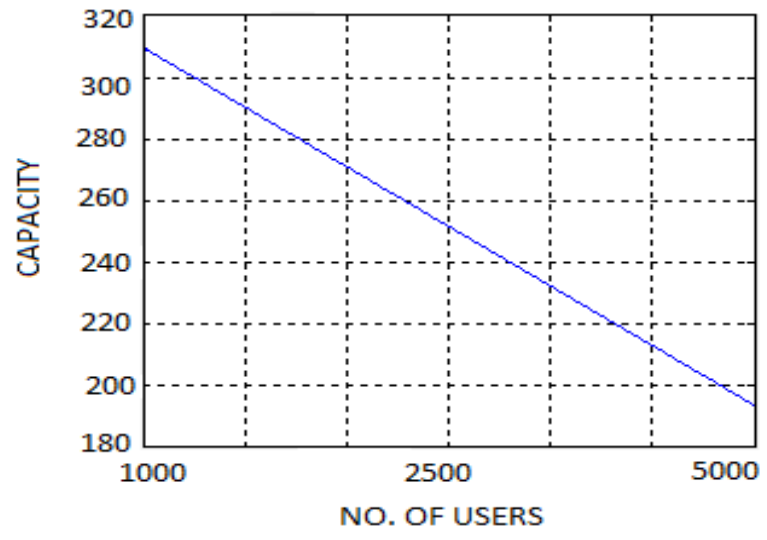

Fig 12: Capacity of ROF in Ethernet network

Figure 12 shows that capacity is inversely proportional to no. of users when ROF deployed as a backhaul technology in Ethernet network.

So, both graphs represent the relationship between capacity on $y$-axis and no. of users on x-axis. In both backhaul technologies the values of capacity decreases from higher values to lower values but radio over fiber performs better as compared to traditional fiber when implemented as backbone technology in Ethernet network.

Table 1: Result based proposition for cellular \& Ethernet

\begin{tabular}{|c|c|c|}
\hline \multicolumn{3}{|c|}{ environment } \\
Paramater & Cellular & Ethernet \\
\hline $\begin{array}{c}\text { Signal to Noise } \\
\text { Ratio }\end{array}$ & $\begin{array}{c}\text { Radio over } \\
\text { Fiber }\end{array}$ & $\begin{array}{c}\text { Radio over } \\
\text { Fiber }\end{array}$ \\
\hline Capacity & $\begin{array}{c}\text { Radio over } \\
\text { Fiber }\end{array}$ & $\begin{array}{c}\text { Radio over } \\
\text { Fiber }\end{array}$ \\
\hline
\end{tabular}

Table 1 shows the simulated results of comparison between ROF and traditional fiber optics, the results in terms of parameters i.e. signal to noise ratio and capacity stated that ROF gives better performance in both heterogeneous environments i.e. Ethernet environment and cellular environment.

\section{CONCLUSION}

ROF and optical fiber have been evaluated as a backhaul technology in two heterogeneous environments, cellular networks and Ethernet (both wired and wireless) networks. The aim of the research was to determine the prevailing technology in both environments considering two variables i.e. signal to noise ratio and capacity. Both variables were analyzed via simulation to produce concrete evidence as how two different heterogeneous networks perform on the backhaul with ROF and traditional fiber optics. According to the results extracted from simulation, and upon critical analysis of these results, ROF proves to be a better solution in terms of signal to noise ratio and capacity. In the future other heterogeneous networks and several parameters like bit error rate, carrier to noise ratio, power consumption and attenuation can be analyzed on radio over fiber. A prototype of such an implementation will be proposed and design for small scale deployment to observe the behavior of Radio over Fiber in terms of afore mentioned parameters as future works.

\section{REFERENCES}

[1] Pirich, R and Mazurowski J, Engineering of fiber optics infrastructure, IEEE Conference Publications, 2012.

[2] S. Wabnitz. Upgrading of soliton transmission lines by a proper dispersion management, CLEO 97 Summaries of Papers Presented at the Conference on Lasers and Electro-Optics, 1997.

[3] Subodh Ku. Gond and Sai Prasad, Green Antenna and Radio over Fiber technology for a cellular wireless. International journal of Engineering and Technology, Vol. 2, July 2012.

[4] H. B. Kim, Radio over Fiber based network architecture. IEEE proceedings in optoelectronics, Vol.143, October 2005.

[5] M. Garcia Larrode and AMJ. Koonen, Towards a reliable RoF infrastructure for broadband wireless access. IEEE proceedings symposium, 2006.

[6] Valencic, Lebinac, Skendzic A, Developments and Current trends in Ethernet technology. IEEE conference Publications, 2013.

[7] P. Yan and X. Yang, Emerging Wireless LANs, Wireless PANs and Wireless MANs. IEEE John wiley Inc., 2008.

[8] Riegel M, Ethernet services over mobile Wimax IEEE journals and magzines, Vol.46, 2008.

[9] S. Sato. Performance analysis of optimum RAKE rceiver for fast fading in DS-CDMA mobile radio Communication system, Proceedings of Vehicular Technology Conference - VTC VETEC-96, 1996.

[10] R.Karthikeyan and Prakasam, A survey on Radio over Fiber for Wireless broadband access technologies. International journal of computer applications, Vol12, February 2013. 